\title{
Angiostrongilosis abdominal
}

\author{
Abdominal angiostrongylosis
}

\author{
Dany F Rivas Méndez, * Ezequiel M Palmisano M,** \\ Quintín González Contreras, ${ }^{*}$ Giovanni Oliva Catalán*
}

Palabras clave:

Nemátodo,

angiostrongilosis,

abdomen agudo.

Key words:

Nematode, angiostrongylosis, acute abdomen .

\footnotetext{
* Servicio de

Emergencia y Cirugía de Hombres del Hospital Regional de Cuilapa, Santa Rosa, Guatemala. ** Centro de

Especialidades Médicas Arenales, "Granadero Baigorria", Santa Fe, Argentina.
}

Recibido: 06/01/2015 Aceptado: 18/03/2016

\section{RESUMEN}

La angiostrongilosis abdominal es una patología endémica de muchos países tropicales de América provocada por el nemátodo Angiostrongylus costaricensis, y cuyas manifestaciones clínicas pueden simular trastornos frecuentes tales como apendicitis aguda y abdomen agudo; los hallazgos quirúrgicos pueden imitar procesos tumorales abdominales. Se describe el caso de un paciente con angiostrongilosis abdominal, una parasitosis frecuente en Guatemala. Material y métodos: Individuo masculino de 16 años con cuadro de dolor abdominal. Revisión de historia clínica e informe anatomopatológico. Tras la resección de la masa ileocecal encontrada en la cirugía de dicho sujeto, el estudio de patología informó hallazgos compatibles con angiostrongilosis abdominal. Conclusión: La angiostrongilosis abdominal es una patología frecuente, por lo que su diagnóstico debe ser considerado en aquellas personas que habitan o visitan áreas endémicas para este nemátodo.

\section{ABSTRACT}

Abdominal angiostrongylosis is an endemic pathology of many tropical countries caused by the Angiostrongylus costaricensis nematode; its clinical complaints can frecuently mimic other disorders, such as appendicitis and acute abdomen; surgical findings can mimic tumoral disease. Material and methods: A male patient with presence of a mass in cecum and ileon. Review of his clinical record and pathological report. After resection of the ileocecal mass, the piece was sent to pathology, and it was the found be abdominal angiostrongylosis. Conclusion: Abdominal angiostrongylosis is a common abdominal disease; therefore, its diagnosis should be considered in patients who live in, or visit, endemic areas for this nematode.

\section{INTRODUCCIÓN}

$\mathrm{L}_{\mathrm{p}}^{\mathrm{a}}$ a angiostrongilosis es una infección parasitaria producida por dos especies del género Angiostrongylus: Angiostrongylus costaricensis y Angiostrongylus cantonensis, responsables de angiostrongilosis abdominal y meningoencefalitis eosinofílica. Su incidencia es mayor entre los meses de agosto y diciembre. El Angiostrongylus costaricensis es un helminto que parasita a la rata algodonera como reservorio principal. ${ }^{1}$

Son parásitos naturales de roedores que utilizan huéspedes intermedios como babosas, caracoles y otros moluscos, siendo la variedad Phyllocaulis variegatus en la que se han encontrado con más frecuencia. Son considerados causa importante de eosinofilia, aunque, a diferencia de las demás parasitosis eosinofílicas, la gastroenteritis no es frecuente. ${ }^{2}$
Dicha eosinofilia es causada por el parásito, al igual que el granuloma, y es mayor en la región ileocecal. ${ }^{3}$

Los parásitos comprenden cinco estadios previos hasta su madurez. Los adultos viven en el interior de las arterias mesentéricas, principalmente en la región ileocecal; desde allí, producen huevos que salen a la submucosa y mucosa, donde alcanzan el primer estadio larvario, migran hacia la luz intestinal y se eliminan con la materia fecal. Los moluscos que actúan como huéspedes intermedios, principalmente babosas y caracoles, se infectan al ingerir estas larvas, las cuales evolucionan a una forma madura - que constituye el tercer estadio- y se tornan infectantes, lo cual ocurre al ingerir accidentalmente el molusco o alimentos contaminados con secreciones, principalmente verduras (Figura 1). 


\section{Figura 1.}

Ciclo vital de la angiostrongilosis. Tomado de la Red de Helmintología de América Latina y El Caribe, catálogo de enfermedades; Universidad Nacional de la Pampa, Argentina.

\section{Epidemiología}

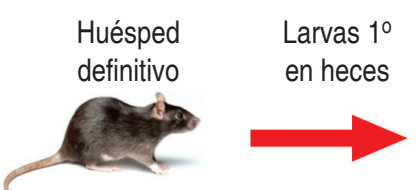

$R$. rattus u otros roedores
La angiostrongilosis abdominal es frecuente en Guatemala y presenta algunas características que consideramos de interés comunicar con el propósito de contribuir al mejor conocimiento de este padecimiento en toda Latinoamérica. En algunos estudios se ha informado que el uso del mebendazole no está contraindicado como antihelmíntico, ya que no exacerba la morbilidad ni la migración parasitaria. ${ }^{4}$ De acuerdo con investigaciones, la mayor frecuencia ocurre entre los meses de agosto y diciembre. ${ }^{5}$

\section{PRESENTACIÓN DEL CASO}

Paciente masculino de 16 años que acudió a consulta por dolor abdominal en la fosa iliaca derecha iniciado tres días antes, acompañado de náusea y vómitos. El examen abdominal reveló dolor abdominal en la fosa iliaca derecha, con signos de psoas y obturador presentes; los exámenes de laboratorio presentaban un recuento de glóbulos blancos de $12.7 \times 10^{3} / \mathrm{mm}^{3}$ (rango de referencia: 5.9-10 $\times 10^{3} / \mathrm{mm}^{3}$ ), con $81.8 \%$ de neutrófilos (rango de referencia: $50-65 \%$ ) y $10 \%$ de eosinófilos (rango de referencia: 0-5\%).

La placa de rayos $\mathrm{X}$ no reveló ningún signo de patología intestinal (Figura 2).

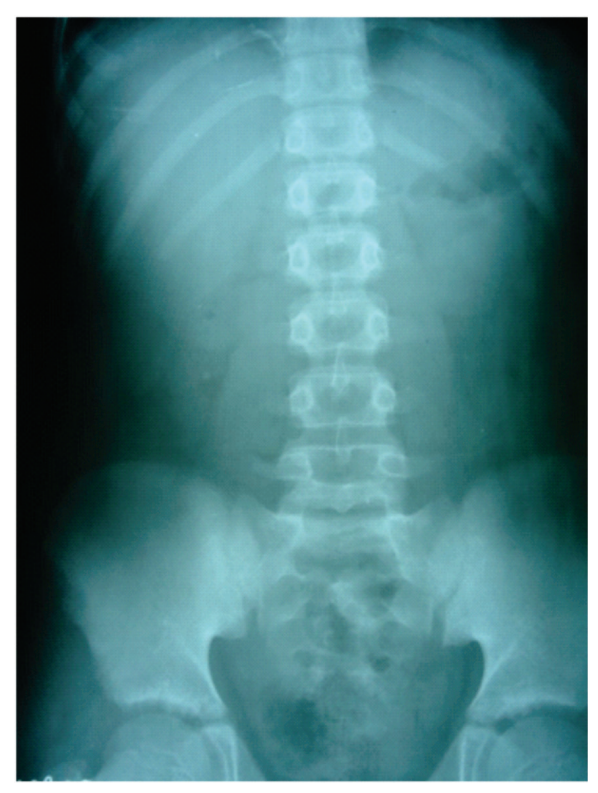

Figura 2. Signos inespecificos en la valoración radiológica del abdomen.

El individuo fue internado con diagnóstico de abdomen agudo quirúrgico, con sospecha de apendicitis aguda, y fue llevado a sala de operaciones, donde se efectuó una incisión de McBurney, con los siguientes hallazgos intrao- 
peratorios: apéndice cecal normal, masa en el ciego (Figura 3) y adenomegalia mesentérica. Se decidió efectuar hemicolectomía derecha y reconstrucción del tránsito mediante una ileotransversoanastomosis terminolateral en dos planos con puntos continuos de polipropileno (Figura 4). Su evolución postoperatoria fue buena; inició tolerancia oral a las 48 horas y fue dado de alta a las 72 horas. El laboratorio de anatomía patológica informó reacción granulomatosa eosinofílica en los cortes histológicos de ciego y de íleon terminal (Figura 5), y se encontraron huevos y larvas de Angiostrongylus costaricensis.

Debido a estos hallazgos, se inició mebendazole a dosis de 100 miligramos cada 12 horas durante tres días.

\section{DISCUSIÓN}

La angiostrongilosis abdominal es una parasitosis endémica de la región suroriente de Guatemala; el nemátodo responsable es el Angiostrongylus costaricensis, ${ }^{1}$ patógeno que parasita al molusco babosa (Vaginulus plebeius) para evolucionar a sus formas infectantes. Ocasiona un cuadro de dolor abdominal en el hombre, que se infecta con la ingestión de los huevos presentes en alimentos contaminados y en las babosas atrapadas, sobre todo en la noche, cuando se ven estos moluscos. ${ }^{6}$

Su afinidad por la región ileocecal provoca una reacción granulomatosa que origina

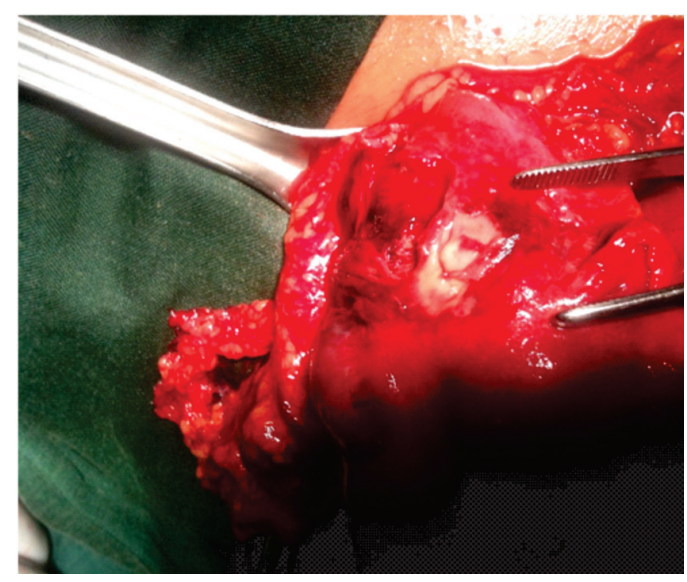

Figura 3. Masa ileocecal y presencia de absceso. cuadros de dolor abdominal, obstrucción e, inclusive, perforación; se han descrito cuadros de peritonitis eosinofílica. Si bien la localización ileocecal es la más frecuente, puede existir afección independiente del íleon terminal, ciego, apéndice o colon ascendente.

Se han descrito dos presentaciones macroscópicas: la pseudoneoplásica — caracterizada por el engrosamiento de la pared intestinal- $y$ la isquémico congestiva - con presencia de lesiones necróticas-.

El tratamiento quirúrgico dependerá de la visualización macroscópica del apéndice y su base en el ciego, así como de la valoración de la adenopatía, ya que algunos manejos quirúrgicos de resecciones amplias se hacen de acuerdo con las múltiples adenopatías encontradas. ${ }^{7} \mathrm{Se}$ han encontrado casos de masas ileocecales en pacientes cuya única manifestación clínica es la hematoquecia. ${ }^{8}$ Se debe tratar según las normas

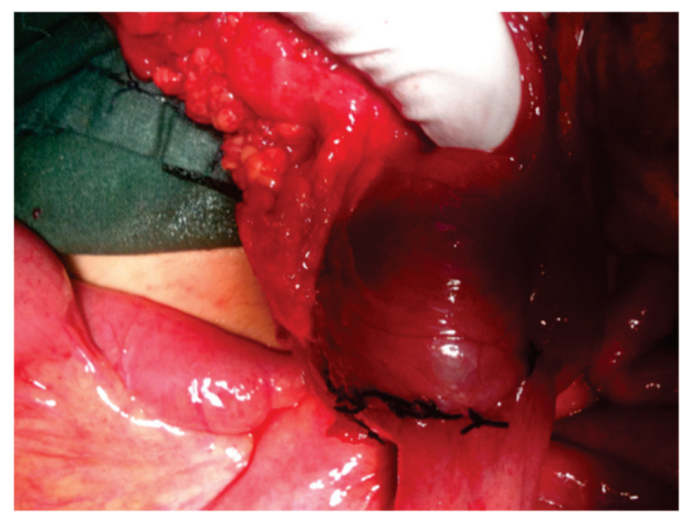

Figura 4. Ileotransversoanastomosis en dos planos.

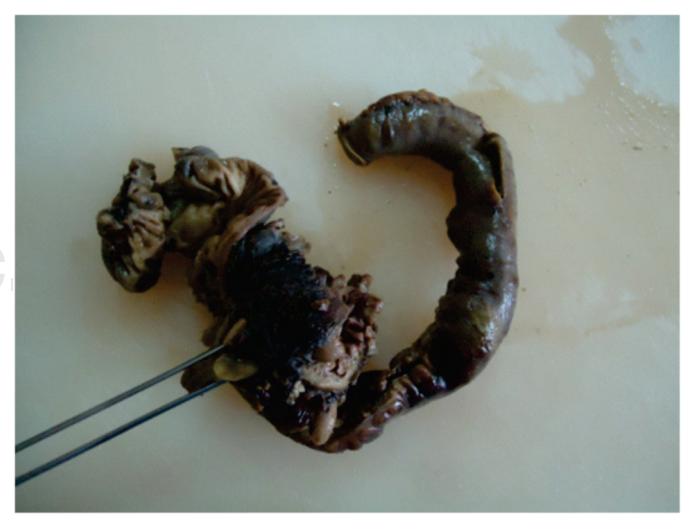

Figura 5. Pieza histopatológica resecada. 
quirúrgicas para neoplasias del apéndice, en donde las masas limitadas al apéndice se tratarán con apendicectomía y las masas por arriba de dos centímetros de diámetro se someterán a hemicolectomía derecha, incluyendo resección apendicular. ${ }^{9}$ Se han descrito localizaciones ectópicas en el hígado y cordón espermático.

Las larvas y el parásito adulto salen a luz intestinal y pueden observarse en el interior de las arteriolas o en los cortes histológicos de tejido que son resecados. La hematología puede estar normal o con leucocitosis y el recuento de eosinófilos puede estar elevado, aunque no debe considerarse patognomónico, ya que existen otras condiciones benignas de colon que pueden provocar eosinofilia, como la colitis y diarrea asociada con eosinofilia. ${ }^{10}$

\section{REFERENCIAS}

1. Botero DM, Restrepo M. Parasitosis humanas para investigaciones biológicas. 4. ${ }^{a}$ ed. Colombia: Corporación para Investigaciones Biológicas; 2005. pp. 323-326.

2. Floch MH. Netter de gastroenterología. España: Elsevier; 2006. pp. 569-571.

3. Hernández de Rodas EC. Epidemiologia de angiostrongiliasis en pacientes con perforación intestinal en hospitales Roosevelt y San Juan de Dios. Guatemala: 2003: 5-6.
4. Mentz MB, Graeff-Teixeira C, Garrido CT. Treatment with mebendazole is not associated with distal migration of adult Angiostrongylus costaricensis in the murine experimental infection. Rev Inst Med Trop Sao Paulo. 2004; 46: 73-75.

5. Loría-Cortés R, Lobo-Sanahuja JF. Clinical abdominal angiostrongylosis. A study of 116 children with intestinal eosinophilic granuloma caused by Angiostrongylus costaricensis. Am J Trop Med Hyg. 1980; 29: 538-544.

6. Rambo PR, Agostini AA, Graeff-Teixeira C. Abdominal angiostrongylosis in southern Brazil — prevalence and parasitic burden in mollusc intermediate hosts from eighteen endemic foci. Mem Inst Oswaldo Cruz. 1997; 92: 9-14.

7. Renzo N, González C. Angiostrongilosis abdominal en Venezuela. Un caso confirmado. Rev Inst Med trop S Paulo. 2007; 49: 197-200.

8. Rodríguez G. Hematoquecia letal por angiostrongilosis abdominal. Biomédica. 2000; 20: 120-125.

9. Takahashi T. Enfermedades médico-quirúrgicas de colon, recto y ano. Capítulo 53. México: Editores Mexicanos Asociados; 2003. pp. 375-382.

10. Wolff B, Beck D. The ASCRS (American Society of Colon and Rectal Surgeons) Textbook of Colon and Rectal Surgery. Capítulo 43. Estados Unidos de América: Springer; 2007. pp. 612-615.

Correspondencia:

Dr. Dany Fernando Rivas Méndez

E-mail: fernandorivas78@hotmail.com 